\title{
TRIGGER TOES
}

\section{Report of a Case}

\section{G. NeEly, Billericay, Essex, England \\ From St Andrew`s Hospital, Billericay}

A man of thirty-seven complained of intermittent painful clawing of the outer four toes of his left foot. His work entailed much climbing up and down steel-runged ladders. He found that when he climbed a ladder the four left outer toes became fixed in a clawed position, which was painful. In order to straighten the clawed toes he had to remove his boot and release them passively by hand, when a click was felt at the medial side of the ankle.

He first noticed this happening three or four years ago. At first only the second toe was involved, but later the third, fourth and fifth toes were also affected. The frequency of the clawing gradually increased until it was happening thirty or forty times a day.

Five years previously the patient had dropped a heavy metal fitting on his left foot, fracturing the necks of the first, second and third metatarsals. There was some consequent flattening of the longitudinal and transverse arches.

Examination confirmed that by flexing his toes the patient could make them lock in a flexed position. The hallux was not affected. He was unable to extend his toes actively. The toes could be extended passively, the movement occurring with a snap and a palpable click behind the medial malleolus, where a thickening on the tendon of the flexor digitorum longus was palpable.

Operation-At exploration the flexor sheath was found thickened at the level of the medial malleolus. When this was divided longitudinally an excess of synovial fluid was found inside. A short length of

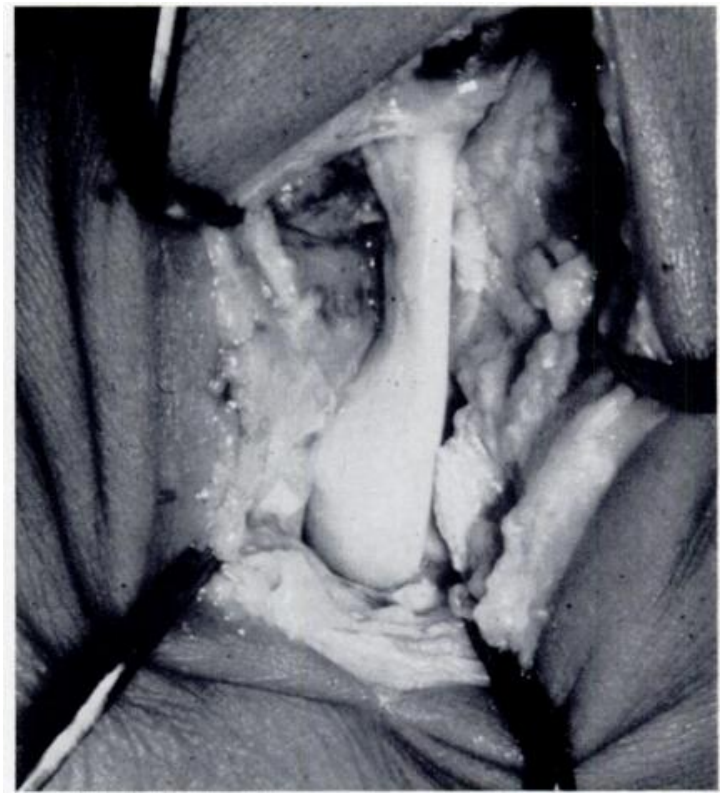

Fig. 1

The exposed tendon of flexor digitorum behind the medial malleolus, showing the narrowed part and the distal thickening. the tendon of flexor digitorum longus was markedly narrowed, and there was a distinct thickening immediately distal to the constricted part (Fig. 1). After the sheath had been divided the toes could be flexed and extended without locking.

Progress - The patient made an uneventful recovery, and when seen several months later was found to be completely relieved.

I wish to thank $\mathrm{Mr} \mathrm{H}$. A. Pearce for permission to publish this_case. 